\title{
Changes of Morphological and Biochemical Properties in Organically Grown Zucchini Squash (Cucurbita pepo L.)
}

\author{
Jasim Uddain \\ Department of Horticulture, Sher-e-Bangla Agricultural University, Dhaka, \\ Bangladesh; and School of Biological Sciences, Universiti Sains Malaysia, \\ 11800 Gelugor, Penang, Malaysia
}

\section{Sanzida Islam Tripti and Mohammad Shah Jahan \\ Department of Horticulture, Sher-e-Bangla Agricultural University, Dhaka, Bangladesh}

\section{Nasrin Sultana \\ Department of Agroforestry \& Environmental Science, Sher-e-Bangla Agricultural University, Dhaka, Bangladesh}

\section{Md Jahedur Rahman \\ Department of Horticulture, Sher-e-Bangla Agricultural University, Dhaka, Bangladesh}

\section{Sreeramanan Subramaniam \\ School of Biological Sciences, Universiti Sains Malaysia, 11800 Gelugor, Penang, Malaysia}

Additional index words. growth, organic farming, pruning, squash, yield

\begin{abstract}
The present research was undertaken with the aim of justifying the effect of morphological, physiological, and nutritional properties in organically produced zucchini squash. The experiment consisted of two factors: three levels of pruning $\left(P_{0}=\right.$ no pruning, $P_{1}=$ primary pruning at 20 days after transplanting $(D A T)$, and $P_{2}=$ secondary pruning at $30 \mathrm{DAT})$ and four levels of vermicompost application $\left(V_{0}=\operatorname{control}, V_{1}=5 \mathrm{t} / \mathrm{ha}, \mathrm{V}_{2}=10 \mathrm{t} / \mathrm{ha}\right.$, and $V_{3}=15 \mathrm{t} / \mathrm{ha}$ ). The results demonstrated that morphological parameters, reproductive components, and yield and proximate compositions were significant differences among the treatments. Increased male and female flower production were recorded from primary pruning with $10 \mathrm{t} / \mathrm{ha}$ vermicompost treatment plots compared with control treatments. Finally, increased fruit number, individual fruit weight, fruit length, fruit diameter, total yield, carbohydrate, protein, crude fat, fiber, and ash were reported from the same treatment combination $\left(\mathrm{P}_{1} \mathrm{~V}_{2}\right)$. Taken together, $10 \mathrm{t} / \mathrm{ha}$ vermicompost with primary pruning appear to provide maximum output in terms of yield and nutrient value compared with other treatments.
\end{abstract}

Squash (Cucurbita pepo L.) is one of the most nutritive and delicious vegetables; its origins are central Mexico, Peru, and the United States (Kathiravan et al., 2006). Squash belongs to the family of Cucurbitaceae and genus of Cucurbita. There are two main types of squash: winter squash and summer squash. Zucchini squash is a summer variety and contains more vitamin $\mathrm{A}$, as well as a significant amount of vitamins $C$, $\mathrm{E}$, and B6; niacin; thiamine; and antioxidant compounds. A $100-\mathrm{g}$ edible portion of zucchini squash contains $94.8 \mathrm{~g}$ of moisture, 0.5 $\mathrm{g}$ of protein, $3.5 \mathrm{~g}$ of carbohydrate, $0.1 \mathrm{~g}$ of

Received for publication 26 Apr. 2019. Accepted for publication 29 May 2019.

J.U. and S.S. are corresponding authors. E-mail: uddain.jasim@gmail.com or sreeramanan@usm.my. fat, $0.8 \mathrm{~g}$ of fiber, $17 \mathrm{kcal}$ of energy, $0.3 \mathrm{~g}$ of minerals, $0.6 \mathrm{mg}$ of iron, $0.02 \mathrm{mg}$ of thiamine, $0.4 \mathrm{mg}$ of niacin, $18 \mathrm{mg}$ of vitamin $\mathrm{C}$, $10 \mathrm{mg}$ of calcium, and $30 \mathrm{mg}$ of phosphorus (Bose et al., 2000). The total vegetable production of Bangladesh is 3365 metric tons (Bangladesh Bureau of Statistics, 2014), but new introduction of squash production is low compared with other vegetables.

Pruning is one of the management practices for squash cultivation that increases the quantity of marketable yield harvested in the first cut, indicating that fruit maturity was also advanced (Devi and Varma, 2014). Varieties of summer squash such as zucchini are often allowed to grow unrestricted on large outdoor plots. Their vines and foliage become long and may curl, giving the impression that much space is necessary to cultivate these vegetables (Esmaielpour and Hokmalipour, 2014). With pruning, the plant takes up less space, and the practice also causes the plant to focus its energy on the remaining fruit, thus growing larger squash. Moreover, pruning helps squash vines grow properly and produce a larger number of squash for harvest.

Excessive use of chemical fertilizers causes pollution of soil, water, and air. There have also been antagonistic effects on human and cattle health due to agrochemicals residue in food products (Kumar and Bohra, 2006). Multiple health hazards and irreparable environmental pollution have occurred from the use of inorganic fertilizers (Hinds et al., 2016) Modern agricultural practices, such as organic or ecological or sustainable agriculture, are being developed to reduce the adverse effects of synthetic fertilizers and pesticides on human health and the environment (Aksoy, 2001; Chowdhury, 2004). Today, researchers have shifted their focus to organic farming to reduce the hazardous effects of chemical fertilizers and pesticides. Recently, consumers have begun to appreciate organic farming due to the enhanced produce quality (Rashid, 2004) Vermicompost is a biocontrol agent that helps to increase the production of crops and protect them from harmful pests without polluting the enbironment (Joshi et al., 2015). Vermicompost also significantly stimulates the growth and productivity of plants (Edwards, 1998) Vermicompost is used as organic fertilizer to improve soil aeration and enhance soil microorganisms, plant growth, and eventually crop yield. Vermicompost also encourages the microbial activity of soil, increases the accessibility of $\mathrm{O}_{2}$, conserves normal soil temperature, improves soil porosity and infiltration of water, increases nutrient content, and ultimately improve growth, yield, and quality of the plant (Arora et al., 2011; Rekha et al., 2018). It also provides plants with essential nutrients and aids in the suppression of plant diseases, and improves root growth and structure (Sarkar et al., 2003).

With all of this in mind, we studied the effects of pruning and vermicomposting on changes of morphophysiological and biochemical properties of zucchini squash.

\section{Materials and Methods}

Experimental site. The research was conducted at the horticultural farm of Sher-eBangla Agricultural University, Dhaka-1207, for two seasons, from Nov. 2015 to Feb. 2016 and from Nov. 2016 to Feb. 2017. The experimental field was located at $90^{\circ} 22^{\prime} \mathrm{E}$ longitude and $23^{\circ} 41^{\prime} \mathrm{N}$ latitude at an altitude of $8.2 \mathrm{~m}$ above the sea level. It was in AgroEcological Zone of Madhupur tract (AEZ No. 28) with deep red-brown terrace soil, which belongs to the Nodda cultivated series. The soil was sandy loam in texture with a particle density of $2.65\left(\mathrm{~g} \cdot \mathrm{ccG}^{-1}\right)$ and bulk density of $1.52\left(\mathrm{~g} \cdot \mathrm{ccG}^{-1}\right)$. The soil $\mathrm{pH}$ was 6.43 ; organic matter $0.84(\%)$; total $\mathrm{N} 0.46 \quad(\%)$; exchangeable K 0.41 (meq/100 g soil); available P 18.65, S 20.92, Fe 225, Zn 4.55, and 
Mg 0.81( $\left.\mu \mathrm{g} \cdot \mathrm{g}^{-1}\right)$, respectively. The experimental area was under the subtropical monsoon climate, which is characterized by scanty rainfall during the Rabi season (November to February).

Planting materials, experimental design, and treatments. The experiment was performed in Randomized Complete Block Design (RCBD) with six replications consisting of two factors: factor $\mathrm{A}$, three levels of pruning $\left(\mathrm{P}_{0}=\right.$ no pruning, $\mathrm{P}_{1}=$ primary pruning at $20 \mathrm{DAT}$, and $\mathrm{P}_{2}=$ secondary pruning at $30 \mathrm{DAT}$ ) and factor $\mathrm{B}$, four levels of vermicompost application $\left(\mathrm{V}_{0}=\right.$ control, $\mathrm{V}_{1}=5 \mathrm{t} / \mathrm{ha}, \mathrm{V}_{2}=10 \mathrm{t} / \mathrm{ha}$, and $\mathrm{V}_{3}=$ $15 \mathrm{t} / \mathrm{ha}$ ). In addition, the treatment combinations were $\mathrm{P}_{0} \mathrm{~V}_{0}, \mathrm{P}_{0} \mathrm{~V}_{1}, \mathrm{P}_{0} \mathrm{~V}_{2}$, and $\mathrm{P}_{0} \mathrm{~V}_{2}, \mathrm{P}_{1} \mathrm{~V}_{0}, \mathrm{P}_{1} \mathrm{~V}_{1}$, $\mathrm{P}_{1} \mathrm{~V}_{2}$, and $\mathrm{P}_{1} \mathrm{~V}_{2}$ and $\mathrm{P}_{2} \mathrm{~V}_{0}, \mathrm{P}_{2} \mathrm{~V}_{1}, \mathrm{P}_{2} \mathrm{~V}_{2}$, and $\mathrm{P}_{2} \mathrm{~V}_{3}$, respectively. Experimental plot size was $2 \mathrm{~m} \times 2 \mathrm{~m}$ and plant spacing was maintained $1 \mathrm{~m}$ $\times 1 \mathrm{~m}$. Each unit plot had four pits and each pit contained one plant.

Seeds sowing, pit preparation, and transplanting of seedlings. Seeds were soaked at $24 \mathrm{~h}$ and then sown in polybags having compost mixed soil for seedling rising. Two seeds were sown in each polybag. The polybags were kept in a shady location. They were watered regularly during the seedling period. When the seedlings $(22 \mathrm{~d}$ old) attained four true leaves and were sufficiently hard, they were transplanted in the pit.
Vermicomposting and pruning. Vermicompost was applied in each plot by making pit as a basal dose. Then expected doses of vermicompost were applied in each pit and covered with one layer of soil for 1 week for composting. Pruning was done according to their treatment. Primary pruning was done by removing the first branch at $20 \mathrm{~d}$ after transplanting, and secondary pruning was done by removing the second branch at $30 \mathrm{~d}$ after transplanting.

Data collection. Growth parameters such as stem diameter $(\mathrm{cm})$, stem length $(\mathrm{cm})$, number of male flowers, number of female flowers, individual fruit weight $(\mathrm{g})$, total number of fruit, fruit length $(\mathrm{cm})$, fruit diameter $(\mathrm{cm})$, yield $(\mathrm{t} / \mathrm{ha})$, and proximate compositions were measured following the standard procedure

Proximate analysis. AOAC (1990) standard methods were used to quantify nutrients for proximate analysis (\% moisture, $\%$ proteins, $\%$ fat, $\%$ ash, and $\%$ carbohydrate) from fruit samples. Oven-drying (Gallenkamp, plus II ovens, Germany) was used to determine moisture content followed by Sadiq et al. (2013). The NCS elementary analyzer (Flash 2000 Organic Elemental Analyzer, Germany) was used to estimate crude proteins. Crude fat content was determined from fruit samples by using hexane as an organic solvent. Ash content was determined by using muffle furnace (Carbolite RHS 1600, Germany), and carbohydrate (\%) was determined with the following formula (Kwiri et al., 2014):

$$
\begin{aligned}
(\%) \mathrm{CHO} & =100-(\% \text { moisture }+ \text { proteins } \\
& +\% \text { Fat }+\% \text { Ash })
\end{aligned}
$$

Statistical analysis. Data were recorded and processed over two growing seasons and analyzed using SPSS statistical software version 21.0. The mean for the treatments was calculated, and analysis of variance for each of the characters was performed by $F$ test. Comparisons of the mean data and SE were determined by DMRT (Duncan's multiple range tests) at $P \leq 0.5$ level of significance.

\section{Results and Discussion}

\section{Effects of growth parameters by pruning} and vermicomposting

Growth parameters were significantly influenced by pruning and vermicompost application. At 40 DAT and 55 DAT, a maximum stem length of $49.5 \mathrm{~cm}$ and $52.3 \mathrm{~cm}$, respectively, was observed with $\mathrm{P}_{2}$ treatment; stem diameter of $1.5 \mathrm{~cm}$ and $2.2 \mathrm{~cm}$, respectively, was recorded with $\mathrm{P}_{1}$ treatment. The shortest stem length with $\mathrm{P}_{1}$ treatment was $47.4 \mathrm{~cm}$ and $51.4 \mathrm{~cm}$, respectively, and stem diameter of $1.4 \mathrm{~cm}$ and $1.9 \mathrm{~cm}$ in $\mathrm{P}_{0}$ (no pruning) treatment (Table 1). Similar results were found during harvest (Table 1).

\begin{tabular}{|c|c|c|c|c|c|c|}
\hline \multirow[b]{2}{*}{ Treatments } & \multicolumn{3}{|c|}{ Stem length $(\mathrm{cm})$} & \multicolumn{3}{|c|}{ Stem diam $(\mathrm{cm})$} \\
\hline & $40 \mathrm{DAT}$ & 55 DAT & Harvest & $40 \mathrm{DAT}$ & 55 DAT & Harvest \\
\hline \multicolumn{7}{|c|}{ Effect of pruning } \\
\hline $\mathrm{P}_{0}$ & $48.6 \pm 1.38 \mathrm{a}$ & $51.5 \pm 1.51 \mathrm{a}$ & $55.1 \pm 1.63 \mathrm{a}$ & $1.2 \pm 0.04 \mathrm{a}$ & $1.4 \pm 0.07 \mathrm{a}$ & $1.9 \pm 0.08 \mathrm{a}$ \\
\hline$P_{1}$ & $47.4 \pm 1.24 \mathrm{a}$ & $51.4 \pm 1.22 \mathrm{a}$ & $55.0 \pm 1.40 \mathrm{a}$ & $1.3 \pm 0.08 \mathrm{a}$ & $1.5 \pm 0.12 \mathrm{a}$ & $2.2 \pm 0.13 \mathrm{a}$ \\
\hline $\mathrm{P}_{2}$ & $49.5 \pm 1.86 \mathrm{a}$ & $52.3 \pm 1.91 \mathrm{a}$ & $55.4 \pm 1.28 \mathrm{a}$ & $1.2 \pm 0.03 \mathrm{a}$ & $1.5 \pm 0.05 \mathrm{a}$ & $2.0 \pm 0.10 \mathrm{a}$ \\
\hline Significance Level & NS & NS & NS & NS & NS & NS \\
\hline \multicolumn{7}{|c|}{ Effect of vermicomposting } \\
\hline $\mathrm{V}_{0}$ & $41.6 \pm 0.65 \mathrm{c}$ & $44.5 \pm 1.11 \mathrm{c}$ & $48.4 \pm 1.62 \mathrm{~b}$ & $1.0 \pm 0.01 \mathrm{~b}$ & $1.2 \pm 0.03 \mathrm{~b}$ & $1.6 \pm 0.04 \mathrm{c}$ \\
\hline $\mathrm{V}_{1}$ & $47.5 \pm 0.71 b$ & $51.4 \pm 0.72 b$ & $56.1 \pm 1.01 \mathrm{a}$ & $1.2 \pm 0.02 \mathrm{~b}$ & $1.3 \pm 0.04 \mathrm{~b}$ & $1.9 \pm 0.07 b$ \\
\hline $\mathrm{V}_{2}$ & $53.1 \pm 1.03 \mathrm{a}$ & $55.9 \pm 1.09 \mathrm{a}$ & $58.7 \pm 1.32 \mathrm{a}$ & $1.4 \pm 0.07 \mathrm{a}$ & $1.7 \pm 0.10 \mathrm{a}$ & $2.3 \pm 0.10 \mathrm{a}$ \\
\hline & $51.9 \pm 0.85 \mathrm{a}$ & $55.1 \pm 0.74 \mathrm{a}$ & $57.5 \pm 1.03 \mathrm{a}$ & $1.4 \pm 0.05 \mathrm{a}$ & $1.6 \pm 0.07 \mathrm{a}$ & $2.3 \pm 0.06 \mathrm{a}$ \\
\hline Significance level & $* * *$ & $* * *$ & $* * *$ & $* * *$ & $* * *$ & $* * *$ \\
\hline
\end{tabular}

Table 1. Effect of pruning and vermicompost on growth parameters of zucchini squash.

Data represent mean \pm SE. Different letters following values within the same column indicate significant effect of pruning and vermicompost at $P \leq 0.05$ and nonsignificant (NS) by Duncan's multiple range tests. DAT $=$ days after transplanting; $\mathrm{P}_{0}=$ no pruning; $\mathrm{P}_{1}=$ primary stem pruning; $\mathrm{P}_{3}=$ secondary stem pruning; $\mathrm{V}_{0}=$ no compost; $\mathrm{V}_{1}=5 \mathrm{t} / \mathrm{ha} ; \mathrm{V}_{2}=10 \mathrm{t} / \mathrm{ha} ; \mathrm{V}_{3}=15 \mathrm{t} / \mathrm{ha}$.

***Highly significant.

\begin{tabular}{|c|c|c|c|c|c|c|}
\hline \multirow[b]{2}{*}{ Treatments } & \multicolumn{3}{|c|}{ Stem length $(\mathrm{cm})$ at } & \multicolumn{3}{|c|}{ Stem diam $(\mathrm{cm})$ at } \\
\hline & $40 \mathrm{DAT}$ & $55 \mathrm{DAT}$ & Harvest & $40 \mathrm{DAT}$ & $55 \mathrm{DAT}$ & Harvest \\
\hline$\overline{\mathrm{P}_{0} \mathrm{~V}_{0}}$ & $41.7 \pm 0.44 \mathrm{~g}$ & $44.0 \pm 1.44 \mathrm{e}$ & $48.3 \pm 3.33 \mathrm{~b}$ & $1.0 \pm 0.01 \mathrm{e}$ & $1.1 \pm 0.01 \mathrm{f}$ & $1.5 \pm 0.04 \mathrm{f}$ \\
\hline $\mathrm{P}_{0} \mathrm{~V}_{1}$ & $48.2 \pm 1.74 \mathrm{def}$ & $52.0 \pm 2.02 \mathrm{bc}$ & $57.4 \pm 2.39 \mathrm{a}$ & $1.1 \pm 0.01 \mathrm{cde}$ & $1.3 \pm 0.03 \mathrm{def}$ & $1.7 \pm 0.06 \mathrm{~d}$-ef \\
\hline $\mathrm{P}_{0} \mathrm{~V}_{2}$ & $52.7 \pm 0.17 \mathrm{abc}$ & $54.3 \pm 0.33 \mathrm{abc}$ & $57.2 \pm 1.09 \mathrm{a}$ & $1.2 \pm 0.03$ bcde & $1.5 \pm 0.15$ bcde & $2.0 \pm 0.09 \mathrm{bcd}$ \\
\hline $\mathrm{P}_{0} \mathrm{~V}_{3}$ & $52.0 \pm 0.50 \mathrm{abcd}$ & $55.5 \pm 1.80 \mathrm{abc}$ & $57.7 \pm 3.03 \mathrm{a}$ & $1.4 \pm 0.12 \mathrm{bc}$ & $1.6 \pm 0.07 \mathrm{~b}$ & $2.2 \pm 0.11 \mathrm{bc}$ \\
\hline$P_{1} V_{0}$ & $41.5 \pm 1.26 \mathrm{~g}$ & $45.8 \pm 2.74 \mathrm{de}$ & $49.3 \pm 4.06 b$ & $1.0 \pm 0.01 \mathrm{e}$ & $1.1 \pm 0.03 \mathrm{cdef}$ & $1.6 \pm 0.12 \mathrm{ef}$ \\
\hline $\mathrm{P}_{1} \mathrm{~V}_{1}$ & $47.8 \pm 1.30$ ef & $51.8 \pm 0.93 \mathrm{bc}$ & $56.8 \pm 1.42 \mathrm{a}$ & $1.1 \pm 0.04 \mathrm{de}$ & $1.3 \pm 0.03 \mathrm{cdef}$ & $2.0 \pm 0.14 \mathrm{bc}$ \\
\hline $\mathrm{P}_{1} \mathrm{~V}_{2}$ & $50.7 \pm 1.74 \mathrm{bcd}$ & $54.3 \pm 1.45 \mathrm{abc}$ & $57.0 \pm 1.53 \mathrm{a}$ & $1.6 \pm 0.11 \mathrm{a}$ & $2.0 \pm 0.14 \mathrm{a}$ & $2.7 \pm 0.06 \mathrm{a}$ \\
\hline $\mathrm{P}_{1} \mathrm{~V}_{3}$ & $49.7 \pm 1.36 \mathrm{cdef}$ & $53.5 \pm 0.29 \mathrm{bc}$ & $56.8 \pm 1.01 \mathrm{a}$ & $1.4 \pm 0.13 \mathrm{ab}$ & $1.6 \pm 0.20 \mathrm{bc}$ & $2.3 \pm 0.16 b$ \\
\hline $\mathrm{P}_{2} \mathrm{~V}_{0}$ & $41.5 \pm 1.80 \mathrm{~g}$ & $43.7 \pm 1.92 \mathrm{e}$ & $47.5 \pm 1.80 \mathrm{~b}$ & $1.0 \pm 0.07 \mathrm{e}$ & $1.2 \pm 0.09$ ef & $1.5 \pm 0.07 \mathrm{f}$ \\
\hline $\mathrm{P}_{2} \mathrm{~V}_{1}$ & $46.5 \pm 0.76 \mathrm{f}$ & $50.3 \pm 0.60 \mathrm{~cd}$ & $54.2 \pm 1.17 \mathrm{ab}$ & $1.2 \pm 0.02 \mathrm{bcde}$ & $1.5 \pm 0.06$ bcde & $1.9 \pm 0.08 \mathrm{cde}$ \\
\hline $\mathrm{P}_{2} \mathrm{~V}_{2}$ & $56.0 \pm 1.53 \mathrm{a}$ & $59.2 \pm 2.05 \mathrm{a}$ & $62.0 \pm 3.06 \mathrm{a}$ & $1.4 \pm 0.05 \mathrm{bc}$ & $1.6 \pm 0.06 \mathrm{bcdd}$ & $2.2 \pm 0.03 \mathrm{~b}$ \\
\hline $\mathrm{P}_{2} \mathrm{~V}_{3}$ & $54.2 \pm 1.20 \mathrm{ab}$ & $56.2 \pm 1.17 \mathrm{ab}$ & $58.0 \pm 1.44 \mathrm{a}$ & $1.3 \pm 0.32 \mathrm{bcd}$ & $1.6 \pm 0.07 \mathrm{bc}$ & $2.3 \pm 0.06 \mathrm{~b}$ \\
\hline Significance level & $* * *$ & $* * *$ & $* * *$ & $* * *$ & $* * *$ & $* * *$ \\
\hline
\end{tabular}

Table 2. Interaction effect of pruning and vermicompost on growth parameters of zucchini squash.

Different letters following mean \pm SE values within the same column indicate significant differences between interaction effects between pruning and vermicompost at $P \leq 0.05$ by Duncan's multiple range tests. $\mathrm{DAT}=$ days after transplanting; $\mathrm{P}_{0}=$ no pruning; $\mathrm{P}_{1}=$ primary stem pruning; $\mathrm{P}_{3}=$ secondary stem pruning; $\mathrm{V}_{0}=$ no compost; $\mathrm{V}_{1}=5 \mathrm{t} / \mathrm{ha} ; \mathrm{V}_{2}=10 \mathrm{t} / \mathrm{ha} ; \mathrm{V}_{3}=15 \mathrm{t} / \mathrm{ha}$.

$* * *$ Highly significant. 
Furthermore, vermicomposting significantly influenced the stem length at various days after transplanting. Compared with the control treatment, the longest stem length $(53.1 \mathrm{~cm})$ and largest stem diameter $(1.7 \mathrm{~cm})$ at 40 DAT were recorded from $\mathrm{V}_{2}$ treatment (Table 1). At 55 DAT and at harvest time, we found similar results with the application of vermicompost (Table 1). The interaction effect of pruning and vermicompost showed statistically significant differences in stem length and diameter. The longest stem length $(56.0 \mathrm{~cm})$ and greatest stem diameter $(1.6 \mathrm{~cm})$ at 40 DAT was observed from the treatment combination $\mathrm{P}_{2} \mathrm{~V}_{2}$ compared with other treatments. Similar results were also found at 55 DAT and harvest time (Table 2).

Pruning helps to generate a better plant condition; sunlight more easily reaches the whole plants, increasing interception of light for photosynthesis. We observed that the stem length and stem of squash were greatest with pruning practices at the early growth stage of $40 \mathrm{~d}$ after transplanting. A similar result was also reported by Syamsi et al. (2017), who noted that proper accumulation of light increases the availability of air circulation and $\mathrm{CO}_{2}$ in the stem and ultimately increases stem length and diameter. The availability of light and $\mathrm{CO}_{2}$ increases the photosynthesis rate in plants, and nonpruned plants display extreme vegetative growth, causing suboptimal use of photosynthesis and resulting in decreases of plant yield (Coggins and Lovatt, 2014). Stem pruning is expected to create optimal space for the vegetative growth, which helps promote photosynthesis, resulting in cell enlargement in fruit length and diameter (Pompelli et al., 2010). Pruning helps reduce unproductive plant parts, which allows the photosynthesis process to be more widely allocated, enhancing fruit weight and production (Yu et al., 2013). We also found that proper pruning significantly enhanced yield-contributing parameters. We observed increased flowering, fruit number per plant, fruit length and diameter, and ultimately increased yield compared with no pruning. Awalin et al. (2017) described a similar result with regard to fruit setting, fruits per plant, and ultimate yield with early-stage stem pruning in bell pepper.

Vermicomposting is an anaerobic and biotic method that promotes eco-friendly, humus-like organic substances (Chanda et al., 2011). Vermicompost is an important organic fertilizer containing high contents of humus, nitrogen ( $2 \%$ to $3 \%)$, phosphorous $(1.50 \%$ to $2.25 \%)$, and potassium $(1.80 \%$ to $2.25 \%)$ micronutrients, as well as beneficial soil microbes such as nitrogen-fixing bacteria and mycorrhiza fungi, which increase vegetative growth of squash (Guerrero 2010). In general, application of vermicompost increases seed germination, stem height, number of leaves, leaf area, leaf dry weight, root length, root number, total yield, number of fruits per plant, chlorophyll content, $\mathrm{pH}$ of juice, total soluble solids of juice, microand macronutrients, carbohydrate (\%), and protein (\%) content and improved the quality of the fruits and seeds (Joshi et al., 2014).

\section{Pruning and vermicompost application} influenced yield attributes

Pruning significantly influenced yieldcontributing parameters in zucchini squash (Tables 3 and 4). The maximum number of female flowers (14.30), number of fruits per plant (10.80), individual fruit weight (313.70 $\mathrm{g})$, fruit length $(17.70 \mathrm{~cm})$, fruit diameter $(5.10$ $\mathrm{P}_{1}$ treatment, but male flowers (11.5) were observed in $\mathrm{P}_{2}$ treatment where the minimum number of female flowers (9.40), number of fruits per plant (7.0), individual fruit weight $(253.30 \mathrm{~g})$, fruit length $(14.80 \mathrm{~cm})$, fruit diameter $(4.50 \mathrm{~cm})$, and yield $(18.90 \mathrm{t} / \mathrm{ha})$ ment (Tables 3 and 4).

Vermicompost also significantly influenced yield-contributing attributes of zucchini squash (Tables 3 and 4). The maximum number of female flowers (13.0), number of fruits per plant (12.3), individual fruit weight $(377.0 \mathrm{~g})$, fruit length $(20.0 \mathrm{~cm})$, fruit diameter $(5.30 \mathrm{~cm})$, and yield $(47.6 \mathrm{t} / \mathrm{ha})$ were recorded from $\mathrm{V}_{2}$ treatment. Only male flower (13.0) was recorded for $\mathrm{V}_{3}$ treatment, whereas the minimum number of female flowers (8.10), number of fruits per plant (5.40), individual fruit weight $(198.60 \mathrm{~g})$, fruit length $(11.10$ $\mathrm{cm})$, fruit diameter $(4.20 \mathrm{~cm})$, and yield $(10.7$ $\mathrm{t} / \mathrm{ha}$ ) (Fig. 1B) were observed in $\mathrm{V}_{0}$ (control) treatment (Tables 3 and 4). $\mathrm{cm})$, and yield (36.30 t/ha) were recorded from (Fig. 1A) was observed in $\mathrm{P}_{0}$ (control) treat-
The combined effect of pruning and vermicompost also influenced yieldcontributing attributes of zucchini squash female flowers (18.70), number of fruits per plant (13.70), individual fruit weight $(433.30 \mathrm{~g})$, fruit length $(21.7 \mathrm{~cm})$, fruit diameter $(5.80 \mathrm{~cm})$, and yield $(59.2 \mathrm{t} / \mathrm{ha})$ were recorded from $\mathrm{P}_{1} \mathrm{~V}_{2}$ treatment. Only male flowers (14.30) were recorded for $\mathrm{P}_{2}$ $\mathrm{V}_{3}$ treatment, whereas the minimum number of female flowers (6.3), number of fruits per plant (4.0), individual fruit weight $(175.30 \mathrm{~g})$, fruit length $(10.30 \mathrm{~cm})$, fruit diameter $(4.10 \mathrm{~cm})$, and yield $(7.0 \mathrm{t} / \mathrm{ha})$ were observed in $\mathrm{P}_{0} \mathrm{~V}_{0}$ (control) treatment (Table 5 and 6).

Vermicomposting is proven to enhance plant growth (Chaoui et al., 2003). We found that vermicomposting significantly influenced the yield and yield-contributing characteristics of zucchini squash. This might be because vermicompost contains a high amount of essential nutrients such as nitrogen, potassium, and micronutrients that enhance photosynthesis, cell division, and cell enlargement, ultimately improving the yield-contributing characteristics of zucchini squash. Joshi et al. (2015) also suggested that vermicompost is an ideal organic manure for better growth and yield of many plants. Jaikishun et al. (2014) reported that vermicomposting improved the micronutrient levels in soil that are important (Tables 5 and 6). The maximum number of

Table 3. Effect of pruning and vermicompost on yield parameters of zucchini squash.

\begin{tabular}{|c|c|c|c|c|}
\hline Treatments & No. male flower & No. female flower & Total no. fruit/plant & Individual fruit wt $(\mathrm{g})$ \\
\hline \multicolumn{5}{|c|}{ Effect of pruning } \\
\hline $\mathrm{P}_{0}$ & $8.5 \pm 0.69 b$ & $9.4 \pm 0.77 \mathrm{~b}$ & $7.0 \pm 0.72 b$ & $253.3 \pm 17.91 \mathrm{~b}$ \\
\hline $\mathrm{P}_{1}$ & $11.0 \pm 0.04 \mathrm{ab}$ & $14.3 \pm 1.16 \mathrm{a}$ & $10.8 \pm 0.88 \mathrm{a}$ & $313.7 \pm 30.46 \mathrm{a}$ \\
\hline $\mathrm{P}_{2}$ & $11.5 \pm 0.96 \mathrm{a}$ & $13.1 \pm 0.97 \mathrm{a}$ & $9.8 \pm 0.87 \mathrm{a}$ & $288.1 \pm 24.52 \mathrm{a}$ \\
\hline Level of significance & $*$ & $* *$ & $* *$ & $* *$ \\
\hline \multicolumn{5}{|c|}{ Effect of vermicomposting } \\
\hline $\mathrm{V}_{0}$ & $6.8 \pm 0.40 \mathrm{c}$ & $8.1 \pm 0.68 \mathrm{c}$ & $5.4 \pm 0.50 \mathrm{c}$ & $198.6 \pm 16.39 \mathrm{c}$ \\
\hline $\mathrm{V}_{1}$ & $9.2 \pm 0.72 b$ & $11.3 \pm 0.86 \mathrm{~b}$ & $8.6 \pm 0.76 b$ & $244.1 \pm 14.25 \mathrm{c}$ \\
\hline $\mathrm{V}_{2}$ & $12.3 \pm 0.94 \mathrm{a}$ & $15.8 \pm 1.15 \mathrm{a}$ & $12.3 \pm 0.72 \mathrm{a}$ & $377.0 \pm 22.77 \mathrm{a}$ \\
\hline $\mathrm{V}_{3}$ & $13.0 \pm 0.83 \mathrm{a}$ & $13.9 \pm 0.90 \mathrm{ab}$ & $10.6 \pm 0.65 \mathrm{a}$ & $320.4 \pm 18.68 b$ \\
\hline Level of significance & $* * *$ & $* * *$ & $* * *$ & $* * *$ \\
\hline
\end{tabular}

Different letters following values within the same column indicate significant differences in pruning and vermicompost at $P \leq 0.05$ by Duncan's multiple range tests. $\mathrm{P}_{0}=$ no pruning; $\mathrm{P}_{1}=$ primary stem pruning; $\mathrm{P}_{3}=$ secondary stem pruning; $\mathrm{V}_{0}=$ no compost; $\mathrm{V}_{1}=5 \mathrm{t} / \mathrm{ha} ; \mathrm{V}_{2}=10 \mathrm{t} / \mathrm{ha} ; \mathrm{V}_{3}=15 \mathrm{t} / \mathrm{ha}$. $* P \leq 0.05 ; * * P \leq 0.01 ; * * *$ highly significant

Table 4. Effect of pruning and vermicomposting on yield parameters of zucchini squash.

\begin{tabular}{lcc}
\hline Treatments & Fruit length $(\mathrm{cm})$ & Fruit diam $(\mathrm{cm})$ \\
\hline $\mathrm{P}_{0}$ & Effect of pruning & $4.5 \pm 0.10 \mathrm{~b}$ \\
$\mathrm{P}_{1}$ & $14.8 \pm 1.02 \mathrm{a}$ & $5.1 \pm 0.18 \mathrm{a}$ \\
$\mathrm{P}_{2}$ & $17.7 \pm 1.22 \mathrm{a}$ & $4.8 \pm 0.15 \mathrm{ab}$ \\
Level of significance & $16.0 \pm 1.08 \mathrm{a}$ & $* *$ \\
& $\mathrm{NS}$ & $4.2 \pm 0.05 \mathrm{c}$ \\
$\mathrm{V}_{0}$ & Effect of vermicomposting & $4.6 \pm 0.09 \mathrm{~b}$ \\
$\mathrm{~V}_{1}$ & $11.1 \pm 0.40 \mathrm{c}$ & $5.3 \pm 0.17 \mathrm{a}$ \\
$\mathrm{V}_{2}$ & $14.81 \pm 0.57 \mathrm{~b}$ & $5.0 \pm 0.16 \mathrm{a}$ \\
$\mathrm{V}_{3}$ & $20.0 \pm 0.67 \mathrm{a}$ & $* * *$ \\
Level of significance & $18.9 \pm 0.65 \mathrm{a}$ & $* *$
\end{tabular}

Different letters within the same column following mean \pm SE values indicate significant differences of pruning and vermicompost at $P \leq 0.05$ and nonsignificant (NS) by Duncan's multiple range tests. $\mathrm{P}_{0}=$ no pruning; $\mathrm{P}_{1}=$ primary stem pruning; $\mathrm{P}_{3}=$ secondary stem pruning; $\mathrm{V}_{0}=$ no compost $\mathrm{V}_{1}=5 \mathrm{t} / \mathrm{ha} ; \mathrm{V}_{2}=10 \mathrm{t} / \mathrm{ha}$; $\mathrm{V}_{3}=15 \mathrm{t} / \mathrm{ha}$

$* * P \leq 0.01 ; * * *$ highly significant; ${ }^{\mathrm{Ns}}$ nonsignificant. 

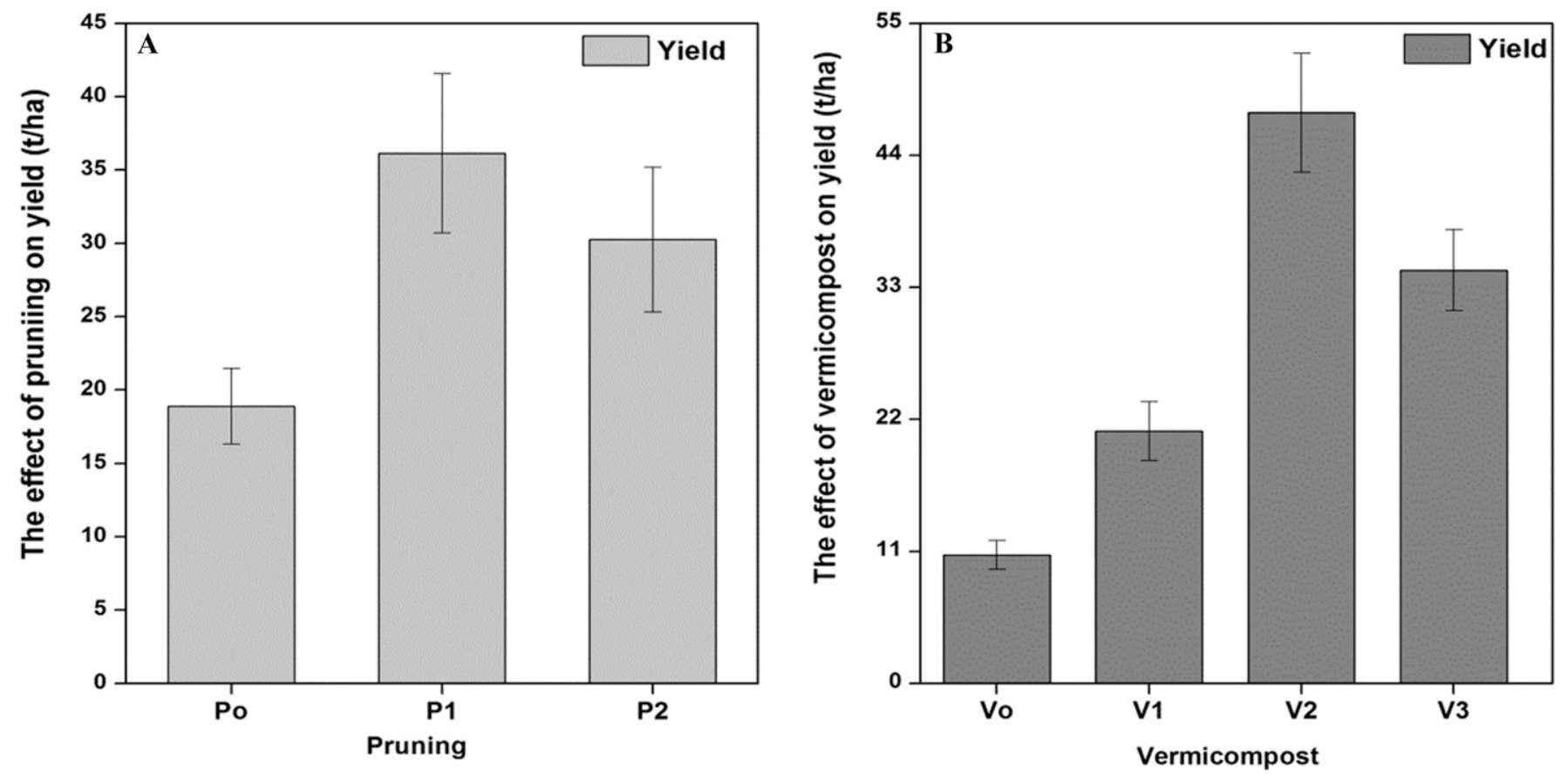

Fig. 1. Effect of (A) pruning and (B) vermicompost on yield (t/ha) of zucchini squash.

Table 5. Combined effects of pruning and vermicompost on yield parameters of zucchini squash.

\begin{tabular}{|c|c|c|c|c|}
\hline Treatments & No. of male flower & No. of female flower & Total no. of fruit/plant & Individual fruit wt $(\mathrm{g})$ \\
\hline$\overline{\mathrm{P}_{0} \mathrm{~V}_{0}}$ & $6.0 \pm 1.00 \mathrm{~d}$ & $6.3 \pm 0.88 \mathrm{~g}$ & $4.0 \pm 0.57 \mathrm{f}$ & $175.3 \pm 2.72 \mathrm{~d}$ \\
\hline $\mathrm{P}_{0} \mathrm{~V}_{1}$ & $7.33 \pm 1.53 \mathrm{bcd}$ & $8.3 \pm 0.66 \mathrm{fg}$ & $6.0 \pm 0.58 \mathrm{e}$ & $258.3 \pm 0.05$ cde \\
\hline $\mathrm{P}_{0} \mathrm{~V}_{2}$ & $9.7 \pm 2.08 \mathrm{bcd}$ & $11.7 \pm 0.89 \mathrm{de}$ & $9.7 \pm 0.88 \mathrm{~cd}$ & $293.3 \pm 3.33 \mathrm{bcd}$ \\
\hline $\mathrm{P}_{0} \mathrm{~V}_{3}$ & $11.0 \pm 1.00 \mathrm{ab}$ & $11.3 \pm 1.20 \mathrm{def}$ & $8.3 \pm 0.88 \mathrm{~d}$ & $286.3 \pm 9.75 \mathrm{~cd}$ \\
\hline $\mathrm{P}_{1} \mathrm{~V}_{0}$ & $7.0 \pm 1.73 \mathrm{~cd}$ & $9.0 \pm 1.15$ efg & $6.3 \pm 0.67 \mathrm{e}$ & $199.0 \pm 4.09 \mathrm{~d}$ \\
\hline $\mathrm{P}_{1} \mathrm{~V}_{1}$ & $9.7 \pm 2.31 \mathrm{bcd}$ & $13.7 \pm 0.88 \mathrm{~cd}$ & $11.0 \pm 0.59 \mathrm{bc}$ & $254.3 \pm 9.19 \mathrm{cde}$ \\
\hline $\mathrm{P}_{1} \mathrm{~V}_{2}$ & $13.7 \pm 2.52 \mathrm{a}$ & $18.7 \pm 1.21 \mathrm{a}$ & $13.7 \pm 0.33 \mathrm{a}$ & $433.3 \pm 6.67 \mathrm{a}$ \\
\hline $\mathrm{P}_{1} \mathrm{~V}_{3}$ & $13.7 \pm 2.89 \mathrm{a}$ & $16.0 \pm 1.0 \mathrm{abc}$ & $12.3 \pm 0.66 \mathrm{ab}$ & $368.0 \pm 9.22 \mathrm{ab}$ \\
\hline $\mathrm{P}_{2} \mathrm{~V}_{0}$ & $7.3 \pm 0.58 \mathrm{bcd}$ & $9.0 \pm 1.0 \mathrm{efg}$ & $6.0 \pm 0.58 \mathrm{e}$ & $221.3 \pm 4.26 \mathrm{de}$ \\
\hline $\mathrm{P}_{2} \mathrm{~V}_{1}$ & $10.7 \pm 1.53 \mathrm{abc}$ & $12.0 \pm 0.58 \mathrm{de}$ & $8.7 \pm 0.33 \mathrm{~d}$ & $219.7 \pm 5.19 \mathrm{de}$ \\
\hline $\mathrm{P}_{2} \mathrm{~V}_{2}$ & $13.7 \pm 2.30 \mathrm{a}$ & $17.0 \pm 0.57 \mathrm{ab}$ & $13.7 \pm 0.33 \mathrm{a}$ & $404.3 \pm 1.73 \mathrm{a}$ \\
\hline $\mathrm{P}_{2} \mathrm{~V}_{3}$ & $14.3 \pm 2.51 \mathrm{a}$ & $14.3 \pm 1.33 \mathrm{bcd}$ & $11.0 \pm 0.00 \mathrm{bc}$ & $307.0 \pm 0.81 \mathrm{bc}$ \\
\hline Level of significance & $* * *$ & $* * *$ & $* * *$ & $* * *$ \\
\hline
\end{tabular}

The data represent mean \pm SE. Different letters within the same column correspond to significant differences at $P \leq 0.05$ by Duncan's multiple range tests. $\mathrm{P}_{0}=$ no pruning; $\mathrm{P}_{1}=$ primary stem pruning; $\mathrm{P}_{3}=$ secondary stem pruning; $\mathrm{V}_{0}=$ no compost; $\mathrm{V}_{1}=5 \mathrm{t} / \mathrm{ha} ; \mathrm{V}_{2}=10 \mathrm{t} / \mathrm{ha} ; \mathrm{V}_{3}=15 \mathrm{t} / \mathrm{ha}$.

$* * *$ Highly significant.

Table 6. Interaction effect of pruning and vermicomposting on yield parameters of Zucchini squash

\begin{tabular}{|c|c|c|c|}
\hline Treatments & Fruit length $(\mathrm{cm})$ & Fruit diam $(\mathrm{cm})$ & Yield (t/ha) \\
\hline $\mathrm{P}_{0} \mathrm{~V}_{0}$ & $10.3 \pm 0.85 \mathrm{~g}$ & $4.1 \pm 0.07 \mathrm{~g}$ & $7.0 \pm 1.24 \mathrm{~g}$ \\
\hline $\mathrm{P}_{0} \mathrm{~V}_{1}$ & $13.2 \pm 0.64 \mathrm{ef}$ & $4.3 \pm 0.13 \mathrm{efg}$ & $15.8 \pm 30.19 \mathrm{f}$ \\
\hline $\mathrm{P}_{0} \mathrm{~V}_{2}$ & $18.4 \pm 0.58 \mathrm{bc}$ & $4.8 \pm 0.09 \mathrm{~cd}$ & $28.4 \pm 2.64 \mathrm{~cd}$ \\
\hline $\mathrm{P}_{0} \mathrm{~V}_{3}$ & $17.4 \pm 0.51 \mathrm{c}$ & $4.6 \pm 0.19 \mathrm{def}$ & $24.4 \pm 4.94 \mathrm{de}$ \\
\hline $\mathrm{P}_{1} \mathrm{~V}_{0}$ & $11.8 \pm 0.80 \mathrm{fg}$ & $4.3 \pm 0.03 \mathrm{efg}$ & $12.0 \pm 1.59 \mathrm{fg}$ \\
\hline $\mathrm{P}_{1} \mathrm{~V}_{1}$ & $16.6 \pm 0.23 \mathrm{~cd}$ & $4.8 \pm 0.03 \mathrm{~cd}$ & $28.2 \pm 4.11 \mathrm{~cd}$ \\
\hline $\mathrm{P}_{1} \mathrm{~V}_{2}$ & $21.7 \pm 0.82 \mathrm{a}$ & $5.8 \pm 0.16 \mathrm{a}$ & $59.2 \pm 2.05 \mathrm{a}$ \\
\hline $\mathrm{P}_{1} \mathrm{~V}_{3}$ & $20.8 \pm 0.73 \mathrm{a}$ & $5.5 \pm 0.21 \mathrm{a}$ & $45.1 \pm 0.44 \mathrm{~b}$ \\
\hline $\mathrm{P}_{2} \mathrm{~V}_{0}$ & $11.2 \pm 0.09 \mathrm{fg}$ & $4.2 \pm 0.05 \mathrm{fg}$ & $13.0 \pm 0.20 \mathrm{fg}$ \\
\hline $\mathrm{P}_{2} \mathrm{~V}_{1}$ & $14.7 \pm 0.68 \mathrm{de}$ & $4.7 \pm 0.14$ cde & $19.1 \pm 1.78 \mathrm{ef}$ \\
\hline $\mathrm{P}_{2} \mathrm{~V}_{2}$ & $19.9 \pm 1.26 \mathrm{ab}$ & $5.4 \pm 0.24 \mathrm{ab}$ & $55.1 \pm 2.02 \mathrm{a}$ \\
\hline $\mathrm{P}_{2} \mathrm{~V}_{3}$ & $18.3 \pm 1.04 \mathrm{bc}$ & $5.1 \pm 0.14 b c$ & $33.8 \pm 2.29 \mathrm{c}$ \\
\hline Level of significance & $* * *$ & $* * *$ & $* * *$ \\
\hline
\end{tabular}

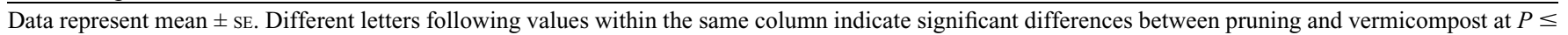

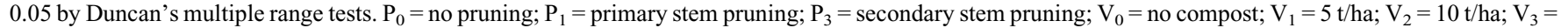
$15 \mathrm{t} /$ ha.

***Highly significant.

for plant growth and development. Vermicomposted soil also contains a high amount of carbon, which supports the slow release of essential nutrients into the soil and allows the plants to utilize available nutrients (Ansari and Sukhraj, 2010). In the present study, we evaluated the effects of vermicomposting on growth and yield of zucchini squash, and significant results were observed with the application of 10 t/ha vermicompost compared with control. The yield was also significantly higher in tomato plants treated with optimal concentrations of 
vermicompost, as suggested by Vaidyanathan and Vijayalakshmi (2017). Azarmi et al. (2008) reported similar results, noting that the addition of optimal quantities of vermicompost significantly increased growth and yield compared with control in tomato plants.

Relationship of different morphological characters of zucchini squash with yield. The yield of zucchini squash was positively correlated (Figs. 2-15) with fruit length $\left(R^{2}=\right.$ $0.8645)$, fruit diameter $\left(R^{2}=0.9448\right)$, number of male flowers $\left(R^{2}=0.776\right)$, number of female flowers $\left(R^{2}=0.9314\right)$, total number of fruits per plant $\left(R^{2}=0.9134\right)$, individual

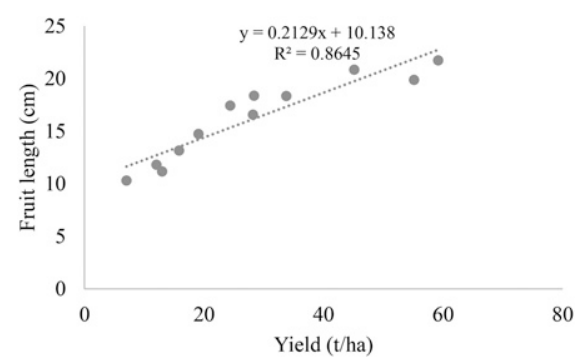

Fig. 2. Relationship between fruit length and yield of zucchini squash.

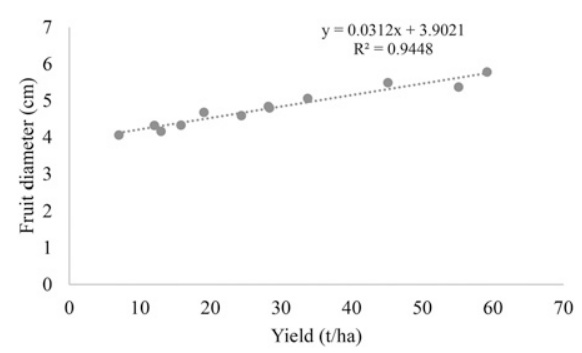

Fig. 3. Relationship between fruit diameter and yield of zucchini squash.

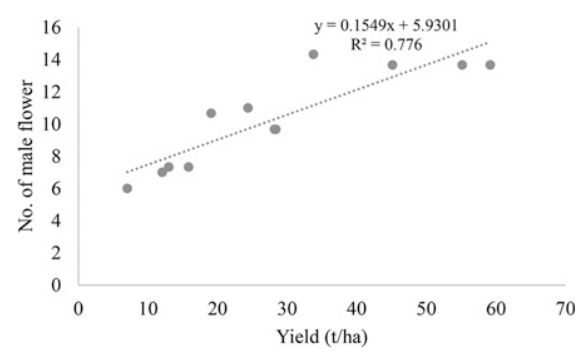

Fig. 4. Relationship between the number of male flower and yield of zucchini squash.

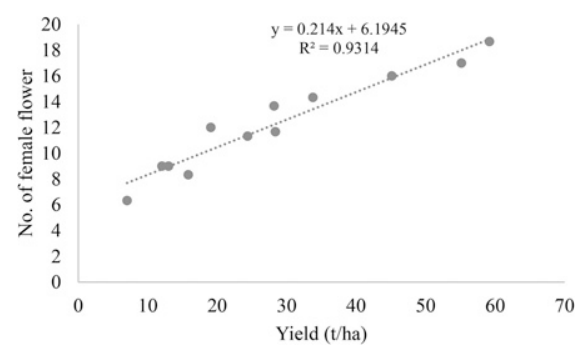

Fig. 5. Relationship between the number of female flower and yield of zucchini squash.

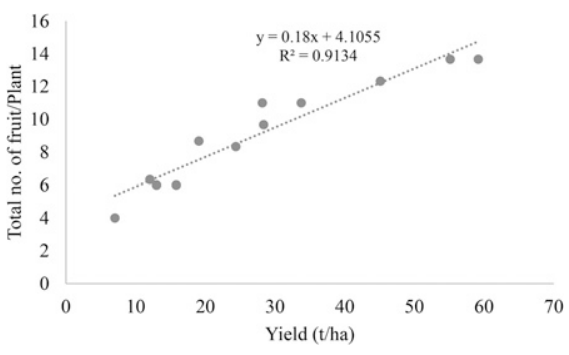

Fig. 6. Relationship between the total number of fruit per plant and yield of zucchini squash.

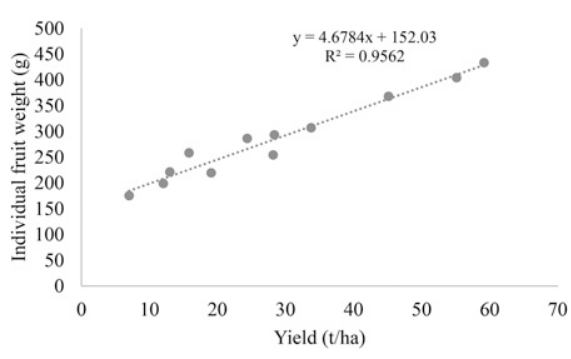

Fig. 7. Relationship between individual fruit weight and yield of zucchini squash.

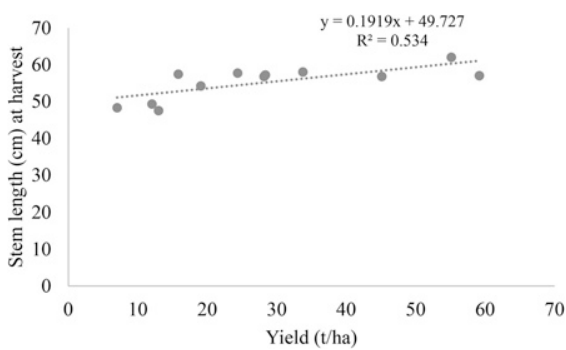

Fig. 8. Relationship between stem length and yield of zucchini squash.

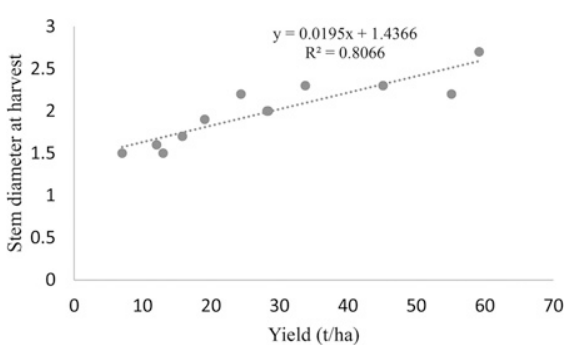

Fig. 9. Relationship between stem diameter and yield of zucchini squash.

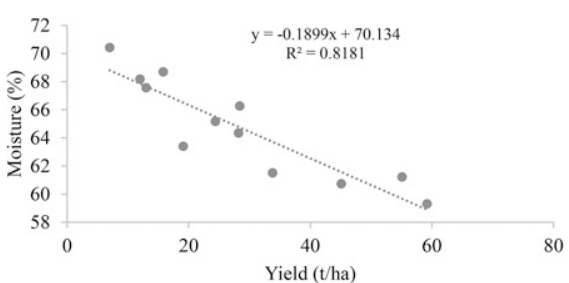

Fig. 10. Relationship between moisture (\%) and yield of zucchini squash.

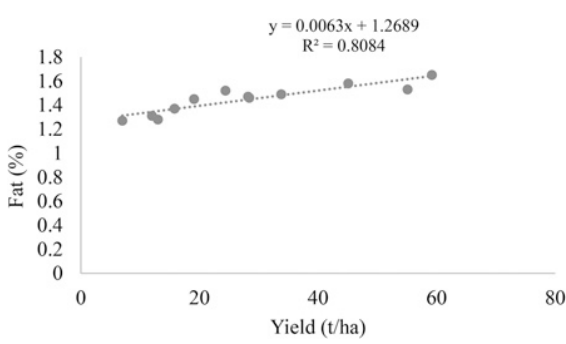

Fig. 11. Relationship between fat (\%) and yield of zucchini squash.

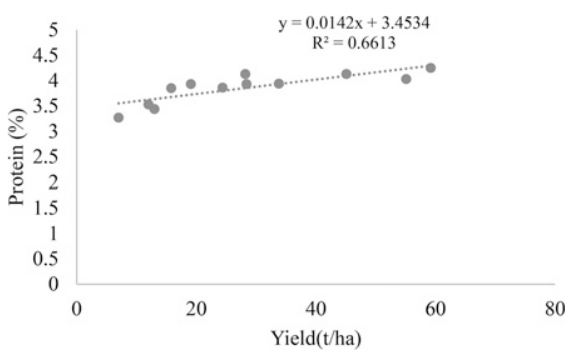

Fig. 12. Relationship between protein (\%) and yield of zucchini squash.

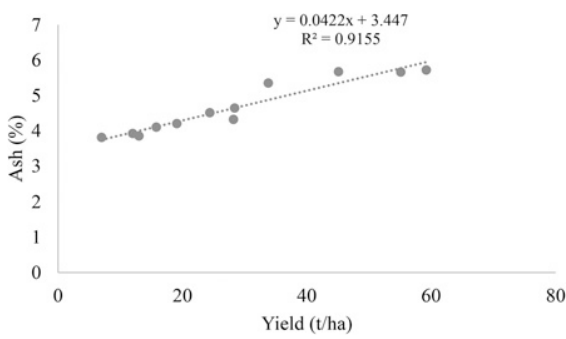

Fig. 13. Relationship between ash (\%) and yield of zucchini squash.

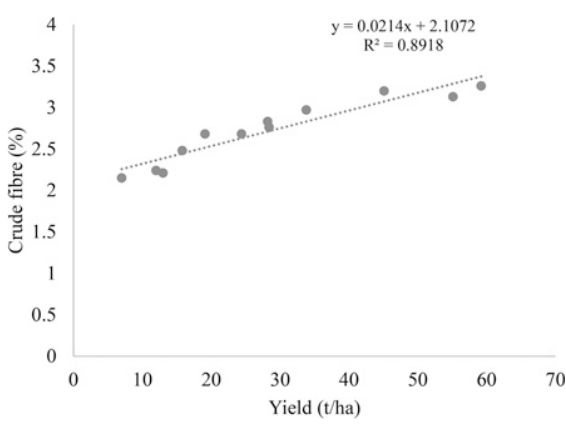

Fig. 14. Relationship between crude fiber (\%) and yield of zucchini squash.

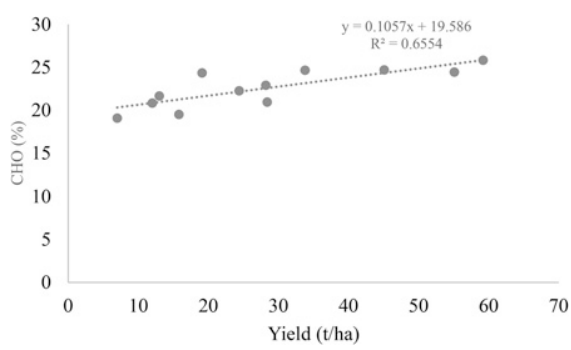

Fig. 15. Relationship between carbohydrate (\%) and yield of zucchini squash. 
Table 7. Effect of pruning and vermicomposting on proximate compositions of zucchini squash.

\begin{tabular}{|c|c|c|c|c|c|c|}
\hline Treatments & Moisture (\%) & Fat $(\%)$ & Protein $(\%)$ & Ash (\%) & Crude fiber $(\%)$ & $\mathrm{CHO}(\%)$ \\
\hline$\overline{\mathrm{P}_{0} \mathrm{~V}_{0}}$ & $70.43 \pm 0.64 \mathrm{a}$ & $1.27 \pm 0.06 \mathrm{c}$ & $3.27 \pm 0.08 \mathrm{c}$ & $3.81 \pm 0.23 \mathrm{c}$ & $2.15 \pm 0.05 \mathrm{e}$ & $19.07 \pm 0.16 \mathrm{f}$ \\
\hline $\mathrm{P}_{0} \mathrm{~V}_{1}$ & $68.70 \pm 0.23 \mathrm{ab}$ & $1.37 \pm 0.08 \mathrm{abc}$ & $3.85 \pm 0.14 b$ & $4.10 \pm 0.24 b c$ & $2.48 \pm 0.08 \mathrm{~d}$ & $19.50 \pm 0.27$ ef \\
\hline $\mathrm{P}_{0} \mathrm{~V}_{2}$ & $66.27 \pm 0.61 \mathrm{bcd}$ & $1.46 \pm 0.10 \mathrm{abc}$ & $3.93 \pm 0.11 b$ & $4.64 \pm 0.26 b$ & $2.76 \pm 0.10 \mathrm{bc}$ & $20.94 \pm 0.89 \mathrm{def}$ \\
\hline $\mathrm{P}_{0} \mathrm{~V}_{3}$ & $65.17 \pm 1.21 \mathrm{cde}$ & $1.52 \pm 0.08 \mathrm{abc}$ & $3.86 \pm 0.10 b$ & $4.51 \pm 0.22 \mathrm{bc}$ & $2.68 \pm 0.09 \mathrm{e}$ & $22.26 \pm 0.82 \mathrm{bcd}$ \\
\hline $\mathrm{P}_{1} \mathrm{~V}_{0}$ & $68.18 \pm 0.58 \mathrm{ab}$ & $1.31 \pm 0.07 \mathrm{bc}$ & $3.53 \pm 0.11 \mathrm{c}$ & $3.92 \pm 0.22 b c$ & $2.24 \pm 0.04 \mathrm{~cd}$ & $20.82 \pm 0.45 \mathrm{def}$ \\
\hline $\mathrm{P}_{1} \mathrm{~V}_{1}$ & $64.35 \pm 2.02 \mathrm{~d}$ & $1.47 \pm 0.07 \mathrm{abc}$ & $4.13 \pm 0.12 \mathrm{ab}$ & $4.32 \pm 0.23 \mathrm{bc}$ & $2.83 \pm 0.05 \mathrm{bc}$ & $22.90 \pm 2.21 \mathrm{bcd}$ \\
\hline $\mathrm{P}_{1} \mathrm{~V}_{2}$ & $59.30 \pm 0.49 \mathrm{~g}$ & $1.65 \pm 0.09 \mathrm{a}$ & $4.25 \pm 0.09 \mathrm{a}$ & $5.72 \pm 0.23 \mathrm{a}$ & $3.26 \pm 0.06 \mathrm{a}$ & $25.82 \pm 0.49 \mathrm{a}$ \\
\hline $\mathrm{P}_{1} \mathrm{~V}_{3}$ & $60.73 \pm 0.86 \mathrm{fg}$ & $1.58 \pm 0.06 \mathrm{ab}$ & $4.13 \pm 0.11 \mathrm{ab}$ & $5.67 \pm 0.26 \mathrm{a}$ & $3.20 \pm 0.07 \mathrm{a}$ & $24.69 \pm 0.67 \mathrm{ab}$ \\
\hline $\mathrm{P}_{2} \mathrm{~V}_{0}$ & $67.57 \pm 0.36 \mathrm{bc}$ & $1.28 \pm 0.09 \mathrm{c}$ & $3.44 \pm 0.10 \mathrm{c}$ & $3.85 \pm 0.22 \mathrm{c}$ & $2.21 \pm 0.07 \mathrm{e}$ & $21.65 \pm 0.84 \mathrm{cdef}$ \\
\hline $\mathrm{P}_{2} \mathrm{~V}_{1}$ & $63.40 \pm 0.61 \mathrm{ef}$ & $1.45 \pm 0.11 \mathrm{abc}$ & $3.93 \pm 0.12 b$ & $4.20 \pm 0.24 b c$ & $2.68 \pm 0.08 \mathrm{~cd}$ & $24.34 \pm 0.54 \mathrm{abc}$ \\
\hline $\mathrm{P}_{2} \mathrm{~V}_{2}$ & $61.22 \pm 0.20 \mathrm{fg}$ & $1.53 \pm 0.08 \mathrm{abc}$ & $4.03 \pm 0.09 \mathrm{ab}$ & $5.66 \pm 0.27 \mathrm{a}$ & $3.13 \pm 0.06 \mathrm{a}$ & $24.43 \pm 0.70 \mathrm{abc}$ \\
\hline $\mathrm{P}_{2} \mathrm{~V}_{3}$ & $61.51 \pm 0.38 \mathrm{fg}$ & $1.49 \pm 0.08 \mathrm{abc}$ & $3.94 \pm 0.07 \mathrm{ab}$ & $5.35 \pm 0.21 \mathrm{a}$ & $2.97 \pm 0.07 \mathrm{ab}$ & $24.65 \pm 0.75 \mathrm{abc}$ \\
\hline Significance level & $* * *$ & NS & $* * *$ & $* * *$ & $* * *$ & $* * *$ \\
\hline
\end{tabular}

Data represent mean \pm SE. Different letters following values within the same column indicate significant differences between pruning and vermicompost at $P \leq$ 0.05 by Duncan's multiple range tests. $\mathrm{CHO}=$ carbohydrate; $\mathrm{NS}=$ nonsignificant; $\mathrm{P}_{0}=$ no pruning; $\mathrm{P}_{1}=$ primary stem pruning; $\mathrm{P}_{3}=$ secondary stem pruning; $\mathrm{V}_{0}=$ no compost; $\mathrm{V}_{1}=5 \mathrm{t} / \mathrm{ha} ; \mathrm{V}_{2}=10 \mathrm{t} / \mathrm{ha} ; \mathrm{V}_{3}=15 \mathrm{t} / \mathrm{ha}$.

***Highly significant; ${ }^{\mathrm{Ns}}$ nonsignificant.

fruit weight $\left(R^{2}=0.9562\right)$, stem length $\left(R^{2}=\right.$ $0.534)$, stem diameter $\left(R^{2}=0.8066\right)$, moisture $\left(R^{2}=0.8181\right)$, fat $\left(R^{2}=0.8084\right)$, protein $\left(R^{2}=0.6613\right)$, ash $\left(R^{2}=0.9155\right)$, crude fiber $\left(R^{2}=0.8918\right)$, and carbohydrate $\left(R^{2}=\right.$ $0.6554)$. These results indicate that with the increase in these characteristics, yield increased as well. Individual fruit weight $\left(R^{2}=0.9562\right)$ had the most intimate relationship with yield, suggesting that squash plant producing higher fruit diameter, number of the female flowers, the total number of fruit, fruit length, stem diameter, and stem length will produce high economic yield and vice versa.

Pruning and vermicompost improved the proximate analysis. Proximate compounds such as carbohydrates $(\%)$, protein $(\%)$, moisture $(\%)$, crude fibers $(\%)$, ash $(\%)$, and fat $(\%)$ were determined from fruit samples in this experiment. Proximate compounds showed significant differences due to pruning and vermicomposting except fat (\%) (Table 7). High content of carbohydrate $(25.82 \%)$, fiber (3.26\%), ash (5.72\%), protein (4.25\%), and fat $(1.65 \%)$ were found from $\mathrm{P}_{1} \mathrm{~V}_{2}$ treatment (primary pruning at 20 DAT with 10 tha vermicompost) whereas lower contents of carbohydrate (19.07\%), fiber (2.15\%), ash (3.81\%), protein $(3.27 \%)$, and fat $(1.27 \%)$ were recorded from the $\mathrm{P}_{0} \mathrm{~V}_{0}$ (control) treatment combination (Table 7). The maximum moisture content $(70.43 \%)$ was seen in the $\mathrm{P}_{0} \mathrm{~V}_{0}$ (control) treatment combination, and the was seen in $\mathrm{P}_{1} \mathrm{~V}_{2}$ treatment (primary pruning at 20 DAT with $10 \mathrm{t} /$ ha vermicompost). Squash is a good energy source because of its high carbohydrate content (Karanja et al., 2014; Sharma and Rao, 2013). Squash has a low content of crude fat, which could be recommended to patients who are overweight as a dietary option and also could be used as a protein source in protein deficiency cases. Adedapo et al. (2009), Elinge et al. (2012), and Kwiri et al. (2014) reported that foods high in fiber help maintain blood cholesterol and anticonstipation. Fiber also helps maintain healthy blood sugar levels and provides energy throughout the day. The presence of high ash content indicates that squash has greater mineral element content, which improves the metabolic process and growth (Elinge et al., 2012; Fedchenkova et al., 2015; Nwofia et al., 2012). We observed similar qualitative properties due to pruning and vermicompost application. We found higher content of carbohydrate, fiber, ash, and protein from $\mathrm{P}_{1} \mathrm{~V}_{2}$ (primary pruning at $20 \mathrm{DAT}$ with $10 \mathrm{t} / \mathrm{ha}$ vermicompost), whereas the lower contents were recorded from $\mathrm{P}_{0} \mathrm{~V}_{0}$ combination (Table 7). The maximum moisture content was determined from $\mathrm{P}_{0} \mathrm{~V}_{0}$ and the minimum level of moisture was counted from $\mathrm{P}_{1} \mathrm{~V}_{2}$ treatment combination. Excess moisture spoilage the fruit rapidly that's why quash fruit need to store at cool conditions (Aruah et al., 2012; Ghani et al., 2013). Ansari and Ismail (2012) reported that vermicomposting facilitates microbial population of $\mathrm{N}_{2}$-fixing bacteria and actinomycetes activities, which are responsible for healthy soil and also help improve quality squash production. The results of our experiment exhibited positive effects not only on growth and yield, but also on some of the elemental content of zucchini squash with the addition of vermicompost compared with untreated control plots. Vermicompost at the optimal concentration increased EC of fruit juice and percentage of fruit dry matter up to $30 \%$ and $24 \%$, respectively, in tomato compared with control in plants (Azarmi et al., 2008).

\section{Conclusion}

In conclusion, the maximum stem length, stem diameter, and flowering and highest fruit number, length and diameter, yield, and proximate properties were observed from the treatment combination of $\mathrm{P}_{1} \mathrm{~V}_{2}$ (primary pruning with $10 \mathrm{t} / \mathrm{ha}$ vermicompost). Therefore, primary pruning with $10 \mathrm{t} / \mathrm{ha}$ vermicompost is a suitable cultural practice for better morphological and biochemical properties of zucchini squash (Cucurbita pepo L.).

\section{Literature Cited}

Adedapo, A.A., O. Florence, J. Anthony, J. Afolayan, and P.J. Masika. 2009. Antioxidant properties of the methanol extracts of the leaves and stems of Celtis africana. Rec. Nat. Prod. 3(1):23-31.

Aksoy, U. 2001. Ecological agriculture: An overview [in Turkish]. Second National
Symposium on Ecological Agriculture, 1416 December, Antalya, Turkey.

Ansari, A.A. and K. Sukhraj. 2010. Effect of vermiwash and vermicompost on soil parameters and productivity of okra (Abelmoschus esculentus) in Guyana. Afr. J. Agr. Res. 5:1794-1798.

Ansari, A.A. and S.A. Ismail. 2012. Role of earthworms in vermitechnology. Agr. Technol. Thail. 8(2):405-415.

AOAC. 1990. Official methods of analysis. 14th ed., p. 76-86. Association of Official Analytical Chemists, Washington, DC.

Arora, V.K., C.B. Singh, A.S. Sidhu, and S.S. Thind. 2011. Irrigation, tillage and mulching effects on soybean yield and water productivity in relation to soil texture. Agr. Water Mgt. 98(4):563-568.

Aruah, B.C., M.I. Uguru, and B.C. Oyiga. 2012. Genetic variability and inter-relationship among some nigerian pumpkin accessions (Cucurbita spp.). Inter. J. Plant Breeding 6(1):34-41

Awalin, S., M. Shahjahan, A.C. Roy, A. Akter, and M.H. Kabir. 2017. Response of bell pepper (Capsicum annuum) to foliar feeding with micronutrients and shoot pruning. J. Agr. Ecol. Res. Intl. 11:1-8.

Azarmi, R., M.T. Giglou, and D. Taleshmikail. 2008. Influence of vermicompost on soil chemical and physical properties in tomato ( $\mathrm{Lyco}$ persicum esculentum) field. African J. Biotechnol. 7(14):2397-2401.

Bangladesh Bureau of Statistics. 2014. The yearbook of agricultural statistics of Bangladesh. Ministry of Planning, Government of the People's Republic of Bangladesh.

Bose, T.K., J. Kabir, P. Das, and P.P. Joy. 2000. Tropical Horticulture, Vol. 1. Naya Prakash, Calcutta.

Chanda, G.C., G. Bhunia, and S.K. Chakraborty. 2011. The effect of vermicompost and other fertilizers on the cultivation of tomato plants. J. Hort. For. 3:42-45.

Chaoui, H.I., L.M. Zibilske, and T. Ohnot. 2003. Effects of earthworms cast and compost on soil microbial activity and plant nutrient availability. Soil Biol. Biochem. 35:295-302.

Chowdhury, R. 2004. Effects of chemical fertilizers on the surrounding environment and the alternative to the chemical fertilizers. Ies. Envis. Newsletter 7(3):4-5.

Coggins, C.W., Jr., and C.J. Lovatt. 2014. Plant growth regulators. In: L. Ferguson and E. Grafton-Cardwell (eds.). Citrus Production 
Manual. University of California Agricultural and Natural Resources, Richmond, CA.

Devi, S. and L.R. Varma. 2014. Quality of muskmelon (Cucumis melo L.) as influenced by plant spacing and levels of pruning under greenhouse. Progress. Hort. 46(1):121-123.

Elinge, C.M., A. Muhammad, F.A. Atiku, Au. Itodo, I.J. Penil, O.M. Sanni, and A.N Mbongo. 2012. Proximate, mineral and antinutrient composition of pumpkin (Cucurbita pepo L.) seeds extract. Intl. J. Plant Res. 2(5):146-150.

Esmaielpour, B. and S. Hokmalipour. 2014. The effects of pruning and potassium nutrition on some morphological traits and seedling properties of pumpkin (Cucurbita pepo L.). Intl. J. Adv. Biol. Biomed. Res. 2(4):1030-1037.

Edwards, C.A. 1998. The use of earthworms in the breakdown and management of organic wastes, p. 327-354. In: Earthworm Ecology. CRC Press, Boca Raton, FL.

Fedchenkova, Y.A., I. Batyuchenko, and O.P. Khvorost. 2015. The study of elemental composition of summer squash (Cucurbita pepo L). ВІСНИК ФАРМАЦІї 1(81):34-37.

Ghani, A., I. Mustafa, A.A. Shah, T. Islam, and I. Ahmed. 2013. Proximate composition of pumpkin (Cucurbita pepo) irrigated with municipal wastewater of Sargodha city. Vegetable Sci. 40(2):159-163.

Guerrero, R.D. 2010. Vermicompost production and its use for crop production in the Philippines. Intl. J. Glob. Environ. Issues 10(3):378-383.

Hinds, J., K.H. Wang, and C.R. Hooks. 2016. Growth and yield of zucchini squash (Cucurbita pepo L.) as influenced by a sunn hemp living mulch. Biol. Agr. Hort. 32(1):21-33.

Jaikishun, S., N. Hunte, A.A. Ansari, and S. Gomathinayagam. 2014. Effect of vermiwash from different sources (Bagasse, Neem, Paddy Straw in different combinations) in controlling fungal diseases and growth of tomato ( $\mathrm{LyCO}$ persicon esculentum) fruits in Guyana. J. Biol. Sci. 14(8):501-507.

Joshi, J., S. Jaswinder, and P.V. Adarsh. 2015 Vermicompost as an effective organic fertilizer and biocontrol agent: Effect on growth, yield, and quality of the plant. Rev. Environ. Sci. Biotechnol. 14:137-159.

Karanja, J.K., B.J. Mugendi, F.M. Khamis, and A.N. Muchugi. 2014. Nutritional Evaluation of Some Kenyan Pumpkins (Cucurbita spp.). Intl. J. Agr. For. 4(3):195-200.

Kathiravan, K., G. Vengedesan, S. Singer, B. Steinitz, H.S. Paris, and V. Gaba. 2006. Adventitious regeneration in vitro occurs across a wide spectrum of squash (Cucurbita pepo) genotypes. Plant Cell Tissue Organ Cult. $85: 285-295$

Kumar, A. and B. Bohra. 2006. Green technology in relation to sustainable agriculture. In: A. Kumar and P. Dubey (eds.). Green technologies for sustainable agriculture. Daya Publishing, Delhi, India.

Kwiri, R., C. Winini, A. Musengi, M. Mudyiwa, C. Nyambi, P. Muredzi, and A. Malunga. 2014. Proximate composition of pumpkin gourd (Cucurbita pepo) seeds from Zimbabwe. Intl. J. Nutr. Food Sci. 3(4):279-283.

Nwofia, G.E., N.N. Victoria, and K.N. Blessing. 2012. Nutritional variation in fruits and seeds of pumpkins (Cucurbita Spp) accessions from Nigeria. Pak. J. Nutr. 11(10):848.

Pompelli, M.F., S.C. Martins, W.C. Antunes, A.R. Chaves, and F.M. DaMatta. 2010. Photosynthesis and photoprotection in coffee leaves are affected by nitrogen and light availabili- ties in winter conditions. J. Plant Physiol. 167(13):1052-1060.

Rashid, M. 2004. Sabji biggan. University Press, Dhaka, Bangledesh.

Rekha, G.S., P.K. Kaleena, D. Elumalai, M.P. Srikumaran, and V.N. Maheswari. 2018. Effects of vermicompost and plant growth enhancers on the exo-morphological features of Capsicum annum (Linn.) pepper. Intl. J. Recycle Org. Waste Agr. 7:83-88.

Sadiq, S., T. Izuagie, M. Shuaibu, M.I. Dogoyaro, A. Garba, and S. Abubakar. 2013. The Nutritional Evaluation and Medicinal Value of Date Palm (Phoenix dactylifera). Intl. J. Modern Chem. 4(3):147-154.

Sarkar, S., S.R. Singh, and R.P. Singh. 2003. The effect of organic and inorganic fertilizer on soil physical condition and the productivity of ricelentil cropping sequence in India. J. Agr. Sci. 140(4):419-425.

Sharma, S. and R. Rao. 2013. Nutritional quality characteristics of pumpkin fruit as revealed by its biochemical analysis. Intl. Food Res. J. 20(5):2309-2316.

Syamsi, M., A.P. Pradana, M. Adiwena, K. Kartina, D. Santoso, R. Wijaya, and A. Maliki. 2017. Effects of pruning on growth and yield of cucumber (Cucumis sativus), ercy variety in the acid soil of north Kalimantan, Indonesia. Cell Biol. Develop. 1:13-17.

Yu, K., Q. Fan, Y. Wang, J. Wei, Q. Ma, D. Yu, and J. Li. 2013. Function of leafy sepals in Paris polyphylla: Photosynthate allocation and partitioning to the fruit and rhizome. Funct. Plant Biol. 40(4):393-399.

Vaidyanathan, G. and A. Vijayalakshmi. 2017. Effect of vermicompost on growth and yield of tomato. Eur. J. Pharm. Med. Res. 4(9):653656. 